\title{
$\kappa$-TOPOLOGIES FOR RIGHT TOPOLOGICAL SEMIGROUPS
}

\author{
JOHN BAKER, NEIL HINDMAN, AND JOHN PYM
}

(Communicated by Johanthan M. Rosenberg)

\begin{abstract}
Given a cardinal $\kappa$ and a right topological semigroup $S$ with topology $\tau$, we consider the new topology obtained by declaring any intersection of at most $\kappa$ members of $\tau$ to be open. Under appropriate hypotheses, we show that this process turns $S$ into a topological semigroup. We also show that under these hypotheses the points of any subsemigroup $T$ with $\operatorname{card} T \leq \kappa$ can be replaced by (new) open sets that algebraically behave like $T$. Examples are given to demonstrate the nontriviality of these results.
\end{abstract}

Let $\kappa$ be a cardinal number. We call a $\kappa$-topology any topology for which the intersection of any family of open sets with no more than $\kappa$ members is again open. Such topologies are easy to come by. If $X$ is any topological space, the sets $V$ of the form $V=\bigcap_{i \in I} U_{i}$ where $\left(U_{i}: i \in I\right)$ is any family of sets open in $X$ with card $I \leq \kappa$ provide a base of open sets for a $\kappa$-topology on $X$. We call this the $\kappa$-topology on $X$, we denote it by $\kappa-X$, we call its members $\kappa$-open sets, and we call $\kappa$-X the $\kappa$-coreflection of $X$.

A semigroup $S$ with a completely regular topology is called right topological if all the maps $s \mapsto s t$ are continuous for $t \in S$. The topological center of $S$ is the subsemigroup

$$
\Lambda(S)=\{s \in S: t \mapsto s t \text { is continuous }\} .
$$

One of our main results is that if $\Lambda(S)$ contains a subset of cardinal $\kappa$ that is dense in $S$ then $\kappa-S$ is a topological semigroup (that is, multiplication is jointly continuous). This theorem allows us to conclude that if $T \subseteq S$ is a subsemigroup, card $T \leq \kappa$ and $U$ is $\kappa$-open with $T \subseteq U$, then there is a $\kappa$-open semigroup $T_{0}$ with $T \subseteq T_{0} \subseteq U$. These results hold in particular for Stone-Čech compactifications of discrete semigroups, the most important of which is $\beta \mathbb{N}$, where $\mathbb{N}$ is the semigroup of positive integers with addition. In the latter case we shall see that the semigroups $T_{0}$ are, in one sense, large.

In the terminology of [3, §2], a space with a $\kappa$-topology is a $P_{\kappa^{+}}$-space. When $\kappa=\aleph_{0}($ as in the case of $\beta \mathbb{N}), \kappa$-topological spaces are more familiar as $P$ spaces (see $[9, \S 1.65])$. The space $\kappa-X$ is then known as the $P$-space coreflection of $X$ [9, Exercise 10B]). It is easy to see that in general $\kappa-X$ is the $\kappa$-coreflection

Received by the editors August 1, 1990.

1991 Mathematics Subject Classification. Primary 22A15.

The second author gratefully acknowledges support received from the National Science Foundation via Grant DMS-8901058. 
of $X$ determined by the following categorical property: the $\kappa$-topology is the finest topology $\tau$ on $X$ such that whenever $Y$ has a $\kappa$-topology and $f: Y \rightarrow X$ is continuous then $f: Y \rightarrow(X, \tau)$ is continuous. In particular, every set open (or closed) in $X$ is also $\kappa$-open (or $\kappa$-closed). The open sets in $\aleph_{0}-X$ are precisely the unions of $G_{\delta}$ 's in $X$. Obviously, if every point of a Hausdorff space $X$ has a basis consisting of not more than $\kappa$ neighborhoods then $\kappa-X$ is discrete. We also remark that if $\kappa$ is finite the $\kappa$-topology is just the original topology.

We shall restrict ourselves to completely regular (Hausdorff) spaces. For such spaces $X$ and each infinite cardinal $\kappa$, every point has a base of open neighborhoods in $\kappa-X$ that are closed sets in $X$ (and so also closed in $\kappa$ $X)$. For let $G=\bigcap_{i \in I} U_{i}$ be any basic open $\kappa$-neighborhood of $x$, with $U_{i}$ open in $X$ and card $I \leq \kappa$. For each $i$, choose inductively a sequence $\left(W_{i}^{n}\right)$ of neighborhoods of $x$ open in $X$ with $W_{i}^{0} \subseteq U_{i}$ and $\operatorname{cl} W_{i}^{n+1} \subseteq W_{i}^{n}$ for $n \geq 0$. Then $\bigcap_{i, n} W_{i}^{n}=\bigcap_{i, n} \mathrm{cl} W_{i}^{n}$ is an open $\kappa$-neighborhood of $x$ (since $\operatorname{card}(I \times \mathbb{N}) \leq \kappa)$ contained in $G$ and is obviously closed in $X$.

We now show that the $\kappa$-topology on a suitable right topological semigroup $S$ has a strong continuity property.

Lemma 1. Let $S$ be a right topological semigroup and let $\kappa$ be infinite. Suppose there is $K \subseteq \Lambda(S)$ such that $K$ is dense in $S$ and card $K \leq \kappa$. Then multiplication is continuous from $S \times \kappa-S$ to $S$.

Proof. Take $a, b \in S$. Let $U$ be any neighborhood of $a b$. Let $U_{0}$ be an open neighborhood of $a b$ with $\mathrm{cl} U_{0} \subseteq U$. Using the fact that $S$ is right topological, find an open neighborhood $V$ of $a$ with $V b \subset U_{0}$. Since $K \subseteq \Lambda(S)$, for each $k \in K \cap V$ we can find an open neighborhood $W_{k}$ of $b$ with $k W_{k} \subseteq U_{0}$. Then $G=\bigcap\left\{W_{k}: k \in K \cap V\right\}$ is a $\kappa$-open neighborhood of $b$. Since $K$ is dense in $S$, it follows that $K \cap V$ is dense in $V$. Therefore if $v \in V$ and $g \in G$, we see that $v g \in \operatorname{cl}(K \cap V) \cdot g \subseteq \operatorname{cl} U_{0} \subseteq U$, again using the fact that $S$ is right topological. Thus $V G \subseteq U$, as required.

Our remaining results are corollaries of Lemma 1.

Theorem 1. Under the hypotheses of Lemma $1, \kappa-S$ is a topological semigroup. Proof. Let $a, b \in S$, and let $E$ be a $\kappa$-neighborhood of $a b$, say $E=\bigcap_{i \in I} U_{i}$ with each $U_{i}$ open in $S$ and card $I \leq \kappa$. For each $i \in I$ use Lemma 1 to find an open neighborhood $V_{i}$ of $a$ and a $\kappa$-neighborhood $G_{i}$ of $b$ with $V_{i} G_{i} \subseteq U_{i}$. Then $F=\bigcap_{i} V_{i}, G=\bigcap_{i} G_{i}$ are $\kappa$-neighborhoods of $a, b$ respectively with $F G \subseteq E$.

We can now prove our theorem about expanding semigroups. It says that, for subsemigroups $T$ that are small enough, the points of $T$ can be replaced by a family of $\kappa$-open sets that algebraically behave like $T$.

Theorem 2. Let $S$ be as in Lemma 1. Let $T \subseteq S$ be a subsemigroup with card $T \leq \kappa$. Let $E$ be a $\kappa$-open set with $T \subseteq E$. Then there is a disjoint family $\{T(t): t \in T\}$ of closed $\kappa$-open subsets of $S$ such that $t \in T(t)$ and $T(s) T(t) \subseteq T(s t)$ for all $s, t \in T$.

Proof. First we produce a disjoint family $\left\{E_{0}(t): t \in T\right\}$ of $\kappa$-open sets with $t \in E_{0}(t) \subseteq E$ for each $t \in T$. For each pair $s, t$ of distinct points of $T$ 
find disjoint open neighborhoods $U_{t}(s), U_{s}(t)$ of $s$ and $t$ respectively. Then $E_{0}(t)=E \cap \bigcap\left\{U_{s}(t): s \in T, s \neq t\right\}$ satisfies our requirements.

The proof is now by induction. For each $n>0$ we find closed (in $S$ ) $\kappa$ open neighborhoods $E_{n}(t)$ of $t$ for each $t \in T$. If $\left\{E_{n}(t): t \in T\right\}$ has been determined, we use Theorem 1 to find $\kappa$-open neighborhoods $F_{n+1}^{t}(s), G_{n+1}^{s}(t)$ with $F_{n+1}^{t}(s) G_{n+1}^{s}(t) \subseteq E_{n}(s t)$; since there is a base for the $\kappa$-topology consisting of closed sets, we may (and do) presume that $F_{n+1}^{t}(s)$ and $G_{n+1}^{s}(t)$ are closed in $S$. Then $E_{n+1}(t)=\bigcap_{s \in T}\left(F_{n+1}^{s}(t) \cap G_{n+1}^{s}(t)\right)$ is a closed $\kappa$-open set containing $t$, and the sets $\left\{E_{n+1}(t): t \in T\right\}$ satisfy $E_{n+1}(s) E_{n+1}(t) \subseteq E_{n}(s t)$ for all $s, t \in T$. Put $T(t)=\bigcap_{n=1}^{\infty} E_{n}(t)$. The family $\{T(t): t \in T\}$ of closed, $\kappa$-open sets is disjoint and satisfies $T(s) T(t) \subseteq T(s t)$ for all $s, t$.

From Theorem 2 we see immediately that $T_{0}=\bigcup_{t \in T} T(t)$ is a semigroup. If we put $\lambda=\operatorname{card} T$ then, being the union of $\lambda$ closed sets, $T_{0}$ is $\lambda$-closed. This establishes the following corollary.

Corollary 1. Let $S, T, E$ be as in Theorem 2 and put $\lambda=\operatorname{card} T$. Then there is a $\lambda$-closed $\kappa$-open semigroup $T_{0}$ with $T \subseteq T_{0} \subseteq E$.

It is worth drawing attention to two special cases of Theorem 2.

Corollary 2. (i) Let $S$ be as in Lemma 1. If $e \in S$ is idempotent and $E$ is a $\kappa$ neighborhood of $e$, there is a closed $\kappa$-open subsemigroup $E_{0}$ with $e \in E_{0} \subseteq E$.

(ii) Let $S$ be as in Lemma 1 and in addition compact. Let $T \subseteq S$ be a finite subsemigroup. Then there is a disjoint family $\{T(t): t \in T\}$ of compact $\kappa$-open subsets of $S$ with $t \in T(t)$ and $T(s) T(t) \subseteq T(s t)$ for all $s, t \in T$.

Corollary 2 (ii) was part of the original inspiration for this paper. It was discovered about 30 years ago that $\beta \mathbb{N}$ is naturally a compact right topological semigroup with an operation + that extends addition in $\mathbb{N}$ and this semigroup has proved to be an invaluable tool in Ramsey Theory (see the surveys $[6,7])$. It was clear from the beginning that $\mathbb{N} \subseteq \Lambda(\beta \mathbb{N})$ (and in fact $\mathbb{N}=\Lambda(\beta \mathbb{N})$, see [4]) and so the conclusions of Theorems 1 and 2 hold for $\beta \mathbb{N}$ with $\kappa=\aleph_{0}$. As remarked above, the $\aleph_{0}$-topology is the $P$-space topology.

Corollary 3. (i) $\beta \mathbb{N}$ is jointly continuous in its $P$-space coreflection topology.

(ii) If $T$ is a countable subsemigroup of $\beta \mathbb{N}$ and $E$ is a $G_{\delta}$ with $T \subseteq E$ then there is a $G_{\delta \sigma}$ subsemigroup $T_{0}$ with $T \subseteq T_{0} \subseteq E$. If $T$ is finite, $T_{0}$ can be chosen to be a compact $G_{\delta}$.

In the case of $\beta \mathbb{N}$ we can add a little more. Each $G_{\delta}$ in $\beta \mathbb{N}$ is large in the sense that it contains a set open in the subspace $\mathbb{N}^{*}=\beta \mathbb{N} \backslash \mathbb{N}[9$, Corollary 3.27]. Thus the semigroup $T_{0}$ is large in $\mathbb{N}^{*}$. In particular, if $G$ is a countable semigroup in $\mathbb{N}^{*}$, then each element $t$ of $G$ can be 'expanded' to a compact set $G(t)$ with nonempty interior in $\mathbb{N}^{*}$ in such a way that $\{G(t): t \in G\}$ is disjoint and satisfies $G(s)+G(t) \subseteq G(s+t)$ for all $s, t \in G$.

The question arises of whether we can arrange for $G(s)+G(t)=G(s+t)$ in this situation. The answer is that equality is never achieved. The reason is that $G(s)+G(t)$ is nowhere dense in $\mathbb{N}^{*}[4$, Theorem 8.1] but $G(s+t)$ has nonempty interior.

If $G$ is a finite group in $\beta \mathbb{N}$ then $G_{0}=\bigcup_{t \in G} G(t)$ is compact subsemigroup with nonempty interior. However, whether $\beta \mathbb{N}$ contains nontrivial finite 
subgroups is unknown (and the question of the existence of such subgroups appears difficult).

The example $\beta \mathbb{N}$ shows that our results do have nontrivial content in at least one interesting case. Obtaining significant examples with $\kappa>\aleph_{0}$ is more difficult.

Example 1. For each cardinal $\kappa$, there exist a compact right topological semigroup $S$ and a subsemigroup $T$ of $S$ for which $T(t)$ is nontrivial for each $t \in T$.

We begin with any infinite discrete semigroup $L$ with $\operatorname{card} L=\kappa$. Put $S=\beta L$. Then $S$ can be made a right topological semigroup with $L \subseteq \Lambda(S)$ (see [6] or [7]). Let $U$ be the set of $\kappa$-uniform ultrafilters on $L$, that is $U=$ $\left\{p \in S\right.$ : for all $A \subseteq S$ with card $\left.A<\kappa, p \notin \mathrm{cl}_{S} A\right\}$. By [3, Corollary 7.8(b)], card $U=2^{2^{\kappa}}$. By [3, Corollary 7.8(a)], if $p \in U$ then each neighborhood base of $p$ has cardinal strictly greater than $\kappa$. So if $T$ is a subsemigroup of $S$ generated by a subset of $U$ of cardinal $\kappa$, then for each $t \in T \cap U$ the $\kappa$-open set $T(t)$ consists of more than one point. Now [5, Theorem 2.5] gives conditions under which $U$ is a semigroup, and this holds in particular if $L$ is cancellative (in fact, $U$ is then an ideal [5, Corollary 2.10]). Thus, for cancellative $L$, we have $T \subseteq U$ and our objective is achieved.

For any semigroup $S$ with a Hausdorff topology there is always a cardinal $\kappa$ such that $\kappa-S$ is jointly continuous, for when $\kappa=\operatorname{card} S, \kappa-S$ is discrete. This suggests that we might use the smallest cardinal with this property as a measure of how discontinuous the multiplication of $S$ is. Theorem 2 shows that sometimes a cardinal smaller than card $S$ will do. We now give two examples, one to show that even for semigroups satisfying the conditions of Lemma 1, card $S$ might be necessary. The other shows that a cardinal smaller than the $\kappa$ of Theorem 1 is sometimes sufficient. (Of course, if $S$ is jointly continuous to begin with, then $\kappa=0$ is sufficient, but our example is not even separately continuous and is, we believe, more significant.)

Example 2. (i) Given regular cardinal $\kappa$, there is a semigroup $S$ with card $S=\kappa$ that satisfies the conditions of Lemma 1 but for which $\lambda-S$ is jointly continuous only if $\lambda \geq \kappa$.

Let $\kappa$ be a regular cardinal (which we regard as an ordinal), so that $\kappa=\operatorname{cf} \kappa$. Write the elements of $\bigoplus_{\kappa} \mathbb{Z}$, the direct sum of $\kappa$ copies of $\mathbb{Z}$, as (transfinite) sequences $\left(z_{\alpha}\right)_{\alpha<\kappa}$ with $z_{\alpha}=0$ for all but finitely many $\alpha$. Define a total order on $\bigoplus_{\kappa} \mathbb{Z}$ by $\left(z_{\alpha}\right)<\left(w_{\alpha}\right)$ if and only if $z_{\mu}<w_{\mu}$ where $\mu=\max \left\{\alpha: z_{\alpha} \neq w_{\alpha}\right\}$ (this is a 'reverse' lexicographic order). Then $\bigoplus_{\kappa} \mathbb{Z}$ is a totally ordered group (with the usual operation + ). We obtain $S$ by adjoining to $\bigoplus_{\kappa} \mathbb{Z}$ two further elements $\infty$ and $-\infty$. We extend the order to $S$ by writing $-\infty<x<\infty$ for all $x \in \bigoplus_{\kappa} \mathbb{Z}$, and we extend + by writing $(-\infty)+x=-\infty$ and $\infty+x=\infty$ for all $x \in \bigoplus_{\kappa} \mathbb{Z}$, and $x+(-\infty)=-\infty$, and $x+\infty=\infty$ for all $x \in S$. We give $S$ a topology by declaring each $x \in \bigoplus_{\kappa} \mathbb{Z}$ to be an isolated point and taking the intervals $[-\infty, x)$ to be basic neighborhoods of $-\infty$ and $(x, \infty]$ to be basic neighborhoods of $\infty$ for $x \in \bigoplus_{\kappa} \mathbb{Z}$. Then $S$ is a right topological semigroup and $\Lambda(S)=\bigoplus_{\kappa} \mathbb{Z}$ (if $x \searrow-\infty$ then $\infty+x=\infty \nrightarrow-\infty=\infty+(-\infty)$, so that $\infty \notin \Lambda(S)$; the other properties of $S$ are equally easy to see).

Now if $\lambda<\kappa \quad(=\operatorname{cf} \kappa$ by hypothesis) the intersection of $\lambda$ intervals of the 
form $(x, \infty]$ contains another of this same form. So we see that $\lambda-S=S$. In particular, $\Lambda(\lambda-S) \neq S$, so $\lambda-S$ does not have a continuous multiplication. (In this example, $\kappa-S$ is discrete.)

(ii) Given any uncountable cardinal $\kappa$ there is a semigroup $S$ that satisfies the conditions of Lemma 1 and that has the property that every dense subset of $S$ has cardinal at least $\kappa$, but for which $\aleph_{0}-S$ is jointly continuous.

Let $\kappa$ be an uncountable cardinal. We start with a semigroup $T$ that has an identity 1 and satisfies (a) $\Lambda(T)$ contains a countable subset $Z$ dense in $T$ (and notice that we may take $1 \in Z$ ) and (b) every point of $T$ has a countable neighborhood base. There do exist compact right topological semigroups with these properties that are not topological (for example, the semigroup $S$ used in [1, Example 1], but with the first copy $\mathbb{T}=\left\{e^{i \theta}: 0 \leq \theta<2 \pi\right\}$ of the circle group replaced by $\left\{e^{i n}: n \in \mathbb{Z}\right\}=\mathbb{Z}_{0}$ (say) to give $\mathbb{Z}_{0} \cup \mathbb{T}_{1} \cup \mathbb{T}_{2}$ ).

We consider the direct product $T^{\kappa}$ with the direct product topology. Then $\Lambda\left(T^{\kappa}\right)=\Lambda(T)^{\kappa} \supseteq Z^{\kappa}$. However, we can find a smaller subset of $\Lambda\left(T^{\kappa}\right)$ that is dense in $T^{\kappa}$; this is the direct sum of $\kappa$ copies of $Z$, a subset dense in the direct product, and it has cardinal exactly $\kappa$. Theorem 1 tells us that $\kappa-T^{\kappa}$ has continuous multiplication, but as in (i) the $\kappa$-topology is uninteresting since it is discrete. Moreover, no subset of $T^{\kappa}$ with fewer than $\kappa$ elements is dense.

We shall determine the $\aleph_{0}$-toplogy on $T^{\kappa}$. Let $\left(t_{\alpha}\right)_{\alpha<\kappa}$ be an element of $T^{\kappa}$. For each $\alpha$, let $\left\{U_{n}\left(t_{\alpha}\right): n=1,2 \ldots\right\}$ be a neighborhood base of $t_{\alpha}$ in $T$ with $U_{n}\left(t_{\alpha}\right) \searrow\left\{t_{\alpha}\right\}$. For any finite subset $F$ of $\kappa$ with card $F=r$, we write $V(F)=\prod_{\alpha<\kappa} V_{\alpha}$ where $V_{\alpha}=T$ for $\alpha \notin F, V_{\alpha}=U_{r}\left(t_{\alpha}\right)$ for $\alpha \in F$. If $E$ is any countable set of predecessors of $\kappa$, we write

$$
W(E)=\bigcap\{V(F): F \text { is a finite subset of } E\} .
$$

Then $W(E)$ is an $\aleph_{0}$-neighborhood of $\left(t_{\alpha}\right)$. It is easy to see that in fact $W(E)=\prod_{\alpha} W_{\alpha}$ where $W_{\alpha}=T$ if $\alpha \notin E, W_{\alpha}=\left\{t_{\alpha}\right\}$ if $\alpha \in E$. It can now be seen that the $\aleph_{0}$-topology on $T$ is determined by neighborhoods of the form $W(E)$.

It is not difficult to check directly that this topology makes multiplication in $T^{\kappa}$ continuous. It is perhaps more illuminating to observe that if $T_{d}$ is $T$ with the discrete topology then $\aleph_{0}-\left(T_{d}\right)^{\kappa}$ is the same as $\aleph_{0}-T^{\kappa}$. Since multiplication in $T_{d}^{\kappa}$ is continuous, so is multiplication in $\aleph_{0}-\left(T_{d}\right)^{\kappa}$ (the argument is as in the proof of Theorem 1).

We conclude with a question. One of the difficult problems about compact right topological semigroups is to determine how the topological and algebraic structures interact. This is even true for minimal one-sided ideals though these have a simple algebraic structure. Thus, for a minimal left ideal $L$, the set $E(L)$ of idempotents in $L$ is a left-zero semigroup $(e f=e$ for all $e, f)$, the semigroups $e L$, for $e \in E(L)$, are isomorphic groups, and algebraically $L$ is isomorphic to the direct product $E(L) \times(e L)[2,1.3 .11,1.2 .16]$. Topologically $L$ is compact. If $S$ has a separately continuous multiplication, then $L$ is isomorphic to the topological direct product of the compact subsemigroups $E(L)$ and $(e L)$ [2, Theorem 1.5.1], but this may not be so in more general cases (see [8] for the semigroup $\beta \mathbb{N}$ ). Theorem 1 tells us that $\kappa-S$ is jointly continuous for some $\kappa$ (though it is not compact); is it true that $\kappa-L$ is isomorphic to a topological direct product of $\kappa-E(L)$ and $\kappa-(e L)$ ? 


\section{REFERENCES}

1. J. W. Baker and P. Milnes, The ideal structure of the Stone-Čech compactification of a group, Math. Proc. Cambridge Philos. Soc. 82 (1977), 401-409.

2. J. Berglund, H. D. Junghenn, and P. Milnes, Analysis on semigroups, Wiley, New York, 1989.

3. W. Comfort and S. Negrepontis, The theory of ultrafilters, Springer-Verlag, Berlin, 1974.

4. E. van Douwen, The Čech-Stone compactification of a discrete groupoid, Topology Appl. 39 (1991), 43-60.

5. N. Hindman, The ideal structure of the space of $\kappa$-uniform ultrafilters on a discrete semigroup, Rocky Mountain J. Math. 16 (1986), 685-701.

6. _ _ Ultrafilters and Ramsey theory-an update, Set Theory and its Applications, (V. Steprans and S. Watson, eds.) Lecture Notes in Math., vol 1401, Springer-Verlag, New York, 1989, pp. 97-118.

7. $ـ$ The semigroups $\beta \mathbb{N}$ and its applications to number theory, The Analytical and Topological Theory of Semigroups, (K. H. Hofmann et al, eds.), de Gruyter, Berlin, 1990, pp. 347-360.

8. J. S. Pym, Footnote to a paper of Baker and Milnes, Math. Proc. Cambridge Philos. Soc. 85 (1979), 315.

9. R. C. Walker, The Stone-Čech compactification, Springer-Verlag, Berlin, 1974.

Department of Pure Mathematics, The University, Sheffield S3 7RH, England

(N. Hindman) Mathematics Department, Howard University, Washington, District of Columbia 20059 